\title{
Letter to the editor: Is a reduced duration of post- discharge surgical site infection surveillance really in our best interests?
}

B M Davies (benjamin.davies4@nhs.net) ${ }^{1}$, H C Patel ${ }^{1}$

1. Department of Neurosurgery, Greater Manchester Neuroscience Centre, Salford Royal Foundation Trust (SRFT), Salford, United Kingdom

Davies BM, Patel HC. Letter to the editor: Is a reduced duration of post-discharge surgical site infection surveillance really in our best interests?. Euro Surveill. 2015;20(13): pii=21081. Available online: http://www.eurosurveillance.org/ViewArticle.aspx?Articleld=21081

\section{To the editor:}

In a recent issue of Eurosurveillance, a well-considered study by Koek et al., among other objectives, investigated the impact of reducing the post-discharge surveillance duration on surgical site infection (SSI) incidence [1] The premise for this was the redefinition in the United States (US) of surveillance for surgeries involving implants, to 90 days, compared with the previously accepted one-year end point. The authors indicate that a similar change is expected in Europe and go on to support this redefinition in their conclusion, despite a potential $14 \%$ of SSIs being overlooked.

Our experience at a single neurosurgical centre would be similar. Of the 1,778 procedures including an implant between October 2011 and February 2014, 61 SSIs were identified after one-year follow-up. If follow-up were restricted to 90 days, this number would be reduced by $15 \%$. Equally, the likelihood of developing an SSI significantly drops after this point. Other studies have identified comparable patterns [2].

So, clearly, redefining the end point will reduce the incidence of SSI. In the US, where financial penalties exist for the development of SSI, some will welcome a reduction in its 'reported' incidence. Koek et al. suggest that by shortening the duration of surveillance, there would be greater consistency among centres, allowing for more accurate and real-time inter-centre comparison. Such comparison has allowed individual centres to successfully recognise and respond to relatively high infection rates [3].

However, if our goal is to advance our knowledge and eliminate the problem entirely, is an artificial reduction in SSI incidence in our best interests? SSI is a relatively uncommon problem with a multifactorial aetiology. Research into combative strategies are challenged by the low event rate, demanding and often failing to attain the large sample numbers required to identify individual advances [4,5]. If the goal posts are magically changed to overlook a large number of SSI, are we not further handicapping our efforts to eliminate this significant problem?

\section{Conflict of interest}

None declared.

Authors' contributions

Both authors contributed to the writing of this letter.

References

1. Koek M, Wille J, Isken M, Voss A, van Benthem B. Postdischarge surveillance (PDS) for surgical site infections: a good method is more important than a long duration. Euro Surveill. 2015;20(8):21042. http://dx.doi.org/10.2807/1560-7917. ES2015.20.8.21042 PMID:25742435

2. Dicks KV, Lewis SS, Durkin MJ, Baker AW, Moehring RW, Chen LF, et al. Surveying the surveillance: surgical site infections excluded by the January 2013 updated surveillance definitions. Infect Control Hosp Epidemiol. 2014;35(5):570-3. http://dx.doi. org/10.1086/675837 PMID:24709727

3. Geubbels ELPE, Bakker HG, Houtman P, van Noort-Klaassen MA, Pelk MSJ, Sassen TM, et al. Promoting quality through surveillance of surgical site infections: five prevention success stories. Am J Infect Control. 2004;32(7):424-30. http://dx.doi. org/10.1016/j.ajic.2004.07.001 PMID:15525920

4. Mendes D, Veiga D, Veiga-Filho J, Fonseca F, de Paiva L, Novo $\mathrm{N}$, et al. Application time for postoperative wound dressing following breast augmentation with implants: study protocol for a randomized controlled trial. Trials. 2015;16(1):19. http:// dx.doi.org/10.1186/s13063-014-0529-5 PMID:25623237

5. Sidhwa F, Itani KMF. Skin preparation before surgery: options and evidence. Surg Infect (Larchmt). 2015;16(1):14-23. http:// dx.doi.org/10.1089/sur.2015.010 PMID:25761076 